\title{
BMJ Open Association of adverse childhood experiences with diabetes in adulthood: results of a cross-sectional epidemiological survey in Singapore
}

\author{
Mythily Subramaniam (D) , ${ }^{1,2}$ Edimansyah Abdin, ${ }^{1}$ Janhavi Ajit Vaingankar, ${ }^{1}$ \\ Sherilyn Chang (D) , Rajeswari Sambasivam, ${ }^{1}$ Anitha Jeyagurunathan, ${ }^{1}$ \\ Lee Seng Esmond Seow, ${ }^{1}$ Rob Van Dam, ${ }^{2}$ Wai Leng Chow, ${ }^{3}$ Siow Ann Chong ${ }^{1}$
}

To cite: Subramaniam M, Abdin E, Vaingankar JA, et al. Association of adverse childhood experiences with diabetes in adulthood: results of a crosssectional epidemiological survey in Singapore. BMJ Open 2021;11:e045167. doi:10.1136/ bmjopen-2020-045167

- Prepublication history and additional material for this paper are available online. To view these files, please visit the journal online (http://dx.doi. org/10.1136/bmjopen-2020045167).

Received 24 September 2020 Revised 08 February 2021 Accepted 21 February 2021

Check for updates

(c) Author(s) (or their employer(s)) 2021. Re-use permitted under CC BY-NC. No commercial re-use. See rights and permissions. Published by BMJ.

${ }^{1}$ Research Division, Institute of Mental Health, Singapore ${ }^{2}$ Epidemiology Domain, Saw Swee Hock School of Public Health, Singapore

${ }^{3}$ Epidemiology and Communicable Diseases Division, Ministry of Health, Singapore

Correspondence to Dr Mythily Subramaniam; Mythily@imh.com.sg

\section{ABSTRACT}

Objectives Several studies have linked diabetes mellitus to adverse childhood experiences (ACEs). While a number of studies have examined the association between ACEs and diabetes in Western populations, few have done it in Asian populations. The current study aimed to examine (1) the association between ACEs and diabetes, including the association after age stratification, and (2) the association of comorbid depression, resource use and health-related quality of life (HRQoL) among those with diabetes and ACEs in Singapore.

Settings Participants were surveyed in their homes or any other preferred venue of their choice.

Participants 6126 individuals aged 18 years and above were randomly selected among Singapore residents. Design Cross-sectional nationwide epidemiological study. Results Exposure to any ACE was not associated with increased odds of diabetes; however, those who had experienced parental separation, death or divorce of a parent had higher odds of diabetes. In addition, we observed significant interaction between age and ACEs in relation to odds of diabetes. ACEs were significantly associated with higher odds of diabetes mainly in the younger age group. The prevalence of major depressive disorder was significantly higher among those with diabetes and ACEs than those with diabetes alone (3.7\% and $0.3 \%$ respectively).

Conclusions Efforts to promote regular exercise and healthy lifestyles both in the population and among those with diabetes must continue for the prevention and management of diabetes. The findings emphasise the need to create more awareness of both the prevalence and impact of ACEs among those treating chronic diseases.

\section{INTRODUCTION}

Adverse childhood experiences (ACEs) is a broad construct that includes neglect, abuse as well as household dysfunction resulting from parental mental illness, substance abuse, imprisonment, domestic violence and divorce occurring before a child reaches 18 years of age. ${ }^{1}$ The prevalence of ACEs varies considerably across studies, depending on
Strengths and limitations of this study

- The study reports on the association of adverse childhood experiences (ACEs) with diabetes based on data from a large representative sample of the Singapore population

- Data on ACEs were captured retrospectively, and there is the possibility of recall bias and, given the sensitivity of the questions, there could have been a tendency to under-report the experience.

- The study did not assess the severity, age of onset and duration of ACEs which may have an impact on the development of diabetes.

- The cross-sectional design limits assertions regarding the sequence of causal events, but this was overcome to a significant extent by excluding those with onset of diabetes before 18 years of age.

the population and the methodology. A study from the USA found an overall prevalence of $46 \%,{ }^{2}$ while another study estimated that about $47 \%$ of the population of England had experienced at least one such ACE. ${ }^{3}$ ACEs impact health outcomes across the life course. ${ }^{4}$ These include emergence of mental and physical illnesses, ${ }^{5-7}$ premature death ${ }^{89}$ and higher healthcare use. ${ }^{10} 11$ Studies have attributed the higher healthcare use among those with ACEs to health comorbidities, functional disability, need for complex care coordination and frequent missing of appointments. ${ }^{12} 13$

Diabetes mellitus (subsequently referred to as diabetes) is a chronic metabolic condition with an estimated global prevalence of $9.3 \%$ (463 million people) in 2019 , which is projected to increase to $10.2 \%$ (578 million) by $2030 .{ }^{14}$ The annual direct cost of diabetesthat is, cost of treating and managing the disease and its complications-was calculated to be Int $\$ 825$ billion in $2014 .^{15}$ Risk factors 
commonly identified for type 1 diabetes include genetic susceptibility, viral infections and toxins while type 2 diabetes has been strongly linked with increasing age, increased body mass index (BMI), ethnicity and family history. ${ }^{16}$

Over the years, studies have linked diabetes to ACEs. A systematic review and meta-analysis suggested that ACEs were associated with a significantly elevated risk of type 2 diabetes in adulthood. Participants who had experienced an ACE had a $32 \%$ increased risk of developing type 2 diabetes later in life. Additionally, they found a differential effect of the type of ACE; neglect had the strongest effect, while physical abuse had the least. ${ }^{17}$ Studies have further shown that endorsement of a single ACE may increase the risk of diabetes in adulthood by $11 \%,{ }^{18}$ and this risk may increase twofold when there are more than three ACEs. ${ }^{19} \mathrm{~A}$ meta-analysis by Jakubowski et $a l^{20}$ examined the association of cumulative childhood adversity (index of at least 2 ACEs) on cardiometabolic disease (cardiovascular disease outcomes and metabolic outcomes-diabetes and metabolic syndrome), and it showed a significant effect of cumulative childhood adversity on adult metabolic outcomes.

Suggested pathophysiological mechanisms linking ACEs with diabetes include effects on the hypothalamicpituitary axis ${ }^{21-23}$; metabolic dysregulation, that is, elevated level of triglycerides, free fatty acids, glucose and insulin caused by the chronic stress from ACEs ${ }^{24}$; elevated levels of inflammatory markers ${ }^{25}$; and epigenetic changes. ${ }^{26} 27$ Psychosocial risk factors have also been proposed as a possible mechanism. Those with a history of ACEs are more likely to have health risk behaviours such as cigarette smoking, alcohol misuse, infrequent physical activity and having higher BMIs, ${ }^{28-30}$ all of which can increase the risk of diabetes. Lastly, studies have shown a significant association of ACEs with post-traumatic stress disorder and depression, which in turn are strongly associated with the development of obesity, diabetes and cardiovascular diseases. ${ }^{19} 2431$ However, much less is known about the correlates of ACEs for adults who have diabetes. Although in general exposure to ACEs is associated with increased burden to physical illness such as depression ${ }^{32}$ and lower health-related quality of life (HRQoL), ${ }^{33}$ it is not known how or if these variables are related to ACEs in patients who have diabetes.

Singapore is a multiethnic country located in Southeast Asia. A recent study found that the lifetime prevalence of ACEs in the adult population of Singapore was $63.9 \%$. ACEs were associated with several mental disorders including major depressive disorder (MDD) and alcohol use disorder. ${ }^{34}$ However, the study did not examine the association of ACEs with physical conditions. The prevalence of diabetes in Singapore is higher compared with the global prevalence, with about one in nine Singaporeans $(11.3 \%)$ suffering from the disease. ${ }^{35}$ Diabetes was the seventh leading cause of disability adjusted life years in Singapore in 2017. ${ }^{36}$ While a number of studies have examined the association between ACEs and diabetes in
Western populations, few have done it in Asian populations. It is also not known whether those with diabetes and ACEs are more likely to be associated with mental disorders or increased resource use.

Accordingly, the current study aimed to examine (1) the association between ACEs and diabetes, including the association after age stratification, and (2) the association of comorbid depression, resource use and HRQoL among those with diabetes and ACEs. The authors hypothesised that experiencing ACEs in childhood would be associated with diabetes, and that the association will be attenuated by health risk behaviours, including obesity, smoking and alcohol use. We also hypothesised that those with diabetes and ACEs would be significantly associated with MDD, increased resource use and lower HRQoL.

\section{METHODS}

The current study comprises secondary analysis of data from the Singapore Mental Health Study conducted in 2016 (SMHS 2016). SMHS 2016 was a population-based, psychiatric epidemiological study conducted among Singapore residents. The study has been described in detail in an earlier article. ${ }^{37}$ In brief, a probability sample was randomly selected using a disproportionate stratified sampling design from a national registry of Singaporeans and permanent residents. Residents aged 65 years and above, Malays and Indians were oversampled to ensure that an adequate sample size would be achieved to improve the reliability of estimates for the subgroup analysis. In all, 6126 respondents were interviewed as part of the study with a response rate of $69.5 \% .^{37}$

\section{Study procedures}

Face-to-face interviews were conducted in English, Chinese or Malay language, depending on the preference of the respondents. Respondents comprised those who were 18 years and older, Singapore citizens or permanent residents, living in Singapore in a community dwelling during the field period. Trained interviewers from a survey research company administered the survey using computer-assisted personal interviews.

\section{Questionnaires}

WHO-Composite International Diagnostic Interview (CIDI) V.3.0

Diagnoses of lifetime MDD were generated using the CIDI V.3.0 algorithm for the Diagnostic and Statistical Manual of Mental Disorders, Fourth Edition, criteria. ${ }^{38}$

A modified version of the CIDI checklist of chronic medical disorders was used to establish the prevalence of 18 chronic medical conditions including diabetes. ${ }^{39}$ The question was read as 'I'm going to read to you a list of health problems some people have. Has a doctor ever told you that you have any of the following...' This was followed by a list of chronic medical conditions which were considered prevalent in Singapore's population. Those who gave a positive answer to the question were 
routed to the question 'How old were you when you were first diagnosed with the condition?'

\section{Adverse Childhood Experiences-International Questionnaire (ACE \\ $-I Q)$}

The WHO's international questionnaire was used to establish the prevalence of ACEs in the current study. ${ }^{40}$ The questions from ACE-IQ included in the current study cover physical, sexual and emotional abuse and neglect by parents or caregivers, as well as family dysfunction. Only one question was asked to assess sexual abuse: 'Did someone touch or fondle you in a sexual way when you did not want them to?' All questions about ACEs pertained to the respondents' first 18 years of life, and responses were binary (yes or no) or frequency-based. From these, a dichotomous variable was created to reflect exposure to each ACE type and category (abuse, neglect, household dysfunction and bullying). Given the sensitive nature of the questions, the questionnaire was selfadministered by the respondent using a tablet. For those who were not literate, the items were read out in a neutral manner by the interviewer. Consistent with previous research, ${ }^{41-43}$ the number of ACEs that the respondents reported having experienced was summed into an ACE count (range 0-11) and categorised into four groups for analysis: 0 ACE, 1 ACE, 2 ACEs and 3+ACEs.

\section{Health risk behaviours}

\section{Overweight and obesity}

BMI was calculated as weight in kilogram divided by the square of height in metre; weight and height were selfreported by participants. BMI was coded into underweight $(<18.5)$, normal (18.5-24.9), overweight (25.0-29.9) and obese $(\geq 30)$, consistent with current recommendations. ${ }^{44}$

\section{Alcohol use}

Based on questions from the WHO-CIDI V.3.0 as well as a series of questions created to assess binge drinking, participants were classified as non-drinkers, drinkers but not binge drinkers, and those with binge drinking.

\section{Smoking history}

Participants were asked if they are current smokers, ex-smokers or non-smokers, that is, have never smoked before. They were then classified as never, past and current smokers.

\section{Short Form (SF)-12 Questionnaire}

The SF-12 instrument was used to measure the generic HRQoL. ${ }^{45}$ It measures eight domains of health: physical functioning, role-physical, bodily pain, general health, vitality (VT), social functioning, role-emotional (RE) and mental health $(\mathrm{MH})$. Scores from these domains were used to generate two summary scores: physical component summary (PCS) and mental component summary (MCS). Both summary scores range between 0 and 100, with higher scores indicating better health. Internal consistency reliability for PCS (Cronbach's alpha=0.81) and MCS (Cronbach's alpha=0.82) domain scores were high in the current study.

\section{Resource use}

Information on healthcare resource use was obtained from respondents using an adapted version of the Client Service Receipt Inventory. ${ }^{46}$ The instrument asked whether respondents had accessed specific healthcare resources during the past 3-month period before the time of the interview. This included care from the polyclinic doctor (government primary care doctors), restructured hospital doctors (a public hospital doctor which is wholly owned by the government), private hospital/clinic doctors and hospitalisations.

\section{Sociodemographic questionnaire}

Data on sex, age, ethnicity (Chinese, Malay, Indian and others), marital status (not married, married, divorced/ separated and widowed), educational level (primary and below, secondary, vocational/institute of technical education, preuniversity/ junior college, diploma and university), employment status (employed, unemployed and economically inactive, ie, students, homemakers and retirees) and monthly household income were collected. Monthly household income was calculated as the average pre-tax income in the past 12 months, of all family members living in the same household.

\section{Statistical analysis}

Statistical analyses were carried out using the STATA V.13.0. All estimates were analysed using survey weights to adjust for oversampling, non-response and poststratification according to age and ethnicity distribution between the survey sample and the Singapore adult population in 2014. The results are presented as 'n', which is the frequency of variable observed/endorsed in the sample and ' $\%$ ', which is the adjusted prevalence in the population. Mean and SEs were calculated for continuous variables and frequencies and percentages for categorical variables. Multiple logistic regression was used to determine sociodemographic factors associated with diabetes, as well as to determine the association of ACEs (specific experiences and counts) with diabetes (model 1), after adjusting for health risk behaviour factors (model 2) and adjusting for both health risk behaviours and sociodemographic factors (model 3). In addition, we tested for interaction between age (18-49 years, and 50+ years) and different ACEs (yes/no) in relation to diabetes by including multiplicative interaction terms in the multivariable logistic regression models. We chose the cut-off age as 49 years as the median age of onset of diabetes in the sample was 49 years. Since the interaction between age and several ACEs was significant, we described the associations between ACEs and diabetes stratified for age.

Multiple linear and logistic regression models were also used to examine the associations among those with diabetes and any ACE (vs among those with diabetes alone) and MDD, primary care visits, restructured hospital doctor visits, private hospital doctor visits, and number of 
hospitalisations after adjusting for health risk behaviour factors and sociodemographic factors.

Those with onset of diabetes up to the age of 18 years were excluded from these analyses as ACEs measure adversities experienced until 18 years. All statistically significant differences were evaluated at the 0.05 level using two-sided tests.

\section{Patient and public involvement}

Patients or participants of this study were not involved in the design or development of the study.

\section{RESULTS}

\section{Sociodemographic characteristics of the sample}

Our analysis on diabetes risk factors included 6106 individuals who responded to the questions on diabetes. Table 1 shows the characteristics of the study population. The sample comprised of equivalent proportion of men $(49.5 \%)$ and women $(50.5 \%)$. The majority of the sample was aged between 18 and 49 years $(60.1 \%)$, of Chinese ethnicity $(75.7 \%)$, married $(59.8 \%)$, with university education $(29.4 \%)$, employed $(72.0 \%)$ and with a monthly household income of Singapore dollars (SGD) 4000 and above $(63.0 \%)$.

\section{Prevalence of diabetes and its correlates}

The prevalence of diabetes in this population was $9.7 \%$ $(\mathrm{n}=904)$. After excluding those with age of onset before 18 years of age, the prevalence of diabetes was $9.5 \%(n=890)$. The mean and median age of onset of diabetes was 48.5 $(\mathrm{SD}=13.5)$ and 49 years, respectively. Older age, Indian ethnicity (vs Chinese), being married (vs single), having preuniversity education (vs university) and being overweight and obese (vs normal BMI) were associated with a greater likelihood of having diabetes. Female gender and having a household income of SGD 6000-9999 (vs less than below SGD 2000) were associated with a lower likelihood of having diabetes (table 1).

\section{Diabetes and ACE}

Our analysis on ACE and diabetes included 4441 participants who responded to the ACE questions.

Table 2 shows the associations between ACEs and diabetes. In the overall sample, emotional neglect and parental separation, divorce or death of a parent were significantly associated with higher odds of diabetes, while experiencing emotional abuse was associated with lower odds of diabetes. In addition, experiencing one or two ACEs as compared with no ACE was associated with higher odds of diabetes. However, after adjusting for sociodemographic and health risk behaviours, only parental separation, divorce or death of a parent remained significantly associated with higher odds of diabetes $(\mathrm{OR}=1.5,95 \% \mathrm{CI}$ 1.01 to 2.2). We observed significant interactions between ACEs and age group in relation to diabetes for several ACEs. Specifically, the association between 'living with household members who were mentally ill or suicidal', emotional abuse, and bullying and diabetes differed significantly for younger (18-49 years) and older $(\geq 50$ years) participants.

Online supplemental table 1 shows the associations between ACEs and diabetes stratified by age without adjusting for confounders, and adjusting for lifestyle factors in model 1 and model 2, respectively. Table 3 shows the fully adjusted associations between ACEs and diabetes stratified according to age. In the younger age group, parental separation, divorce or death of a parent $(\mathrm{OR}=2.2,95 \% \mathrm{CI} 1.1$ to 4.4), and experiencing three or more ACEs ( $\mathrm{OR}=2.3$, $95 \%$ CI 1.1 to 5.4) were significantly associated with higher odds of diabetes. In addition, the association between physical neglect and diabetes was borderline significant in the younger age group (OR=2.3, 95\% CI 0.9 to 5.9$)$. In the older age group, living with household members who were mentally ill or suicidal $(\mathrm{OR}=0.2,95 \% \mathrm{CI} 0.1$ to 0.6$)$ and emotional abuse $(\mathrm{OR}=0.2,95 \% \mathrm{CI} 0.1$ to 0.5$)$ were significantly associated with lower odds of diabetes.

The age of onset of diabetes was substantially earlier in the younger (18-49 years) age group (median=37 years) than in the older ( $>50$ years) age group (median $=51$ years).

Comorbid depression, resource use and HRQoL domains among those with diabetes and ACE versus those with diabetes alone The prevalence of those with both diabetes and ACE was higher than those with diabetes alone $(5.7 \%(\mathrm{n}=350)$ vs $2.1 \%(\mathrm{n}=137))$. Table 4 shows the associations between combinations of diabetes and ACEs with MDD, resource use and HRQoL domains. The prevalence of MDD was significantly higher among those with diabetes and ACEs than those with diabetes alone $(3.7 \%$ and $0.3 \%$ respectively), and after controlling for sociodemographic and health behaviour factors in multivariate regression models, those with diabetes and ACEs remained more likely to be associated with increased odds of MDD than those with diabetes only ( $\mathrm{OR}=19.7,95 \%$ CI 2.1 to 187.4$)$.

Having diabetes with ACE was associated with lower scores on several domains of quality of life such as VT, $\mathrm{RE}$ and $\mathrm{MH}$, and MCS as compared with diabetes without ACEs. In contrast, diabetes with ACE was associated with higher scores on PCS as compared with diabetes without ACEs.

\section{DISCUSSION}

The current study used nationally representative data from an urban Asian population to examine the association of ACEs with diabetes among those with onset of diabetes after 18 years. Although exposure to any ACE was not associated with increased odds of diabetes, those who had experienced parental separation, death or divorce of a parent, had higher odds of diabetes. In addition, we observed a significant interaction between age and ACEs in relation to the odds of diabetes. Among those belonging to the younger age group $(<50$ years), parental separation, divorce or death of a parent, bullying and experiencing three or more ACEs were significantly 


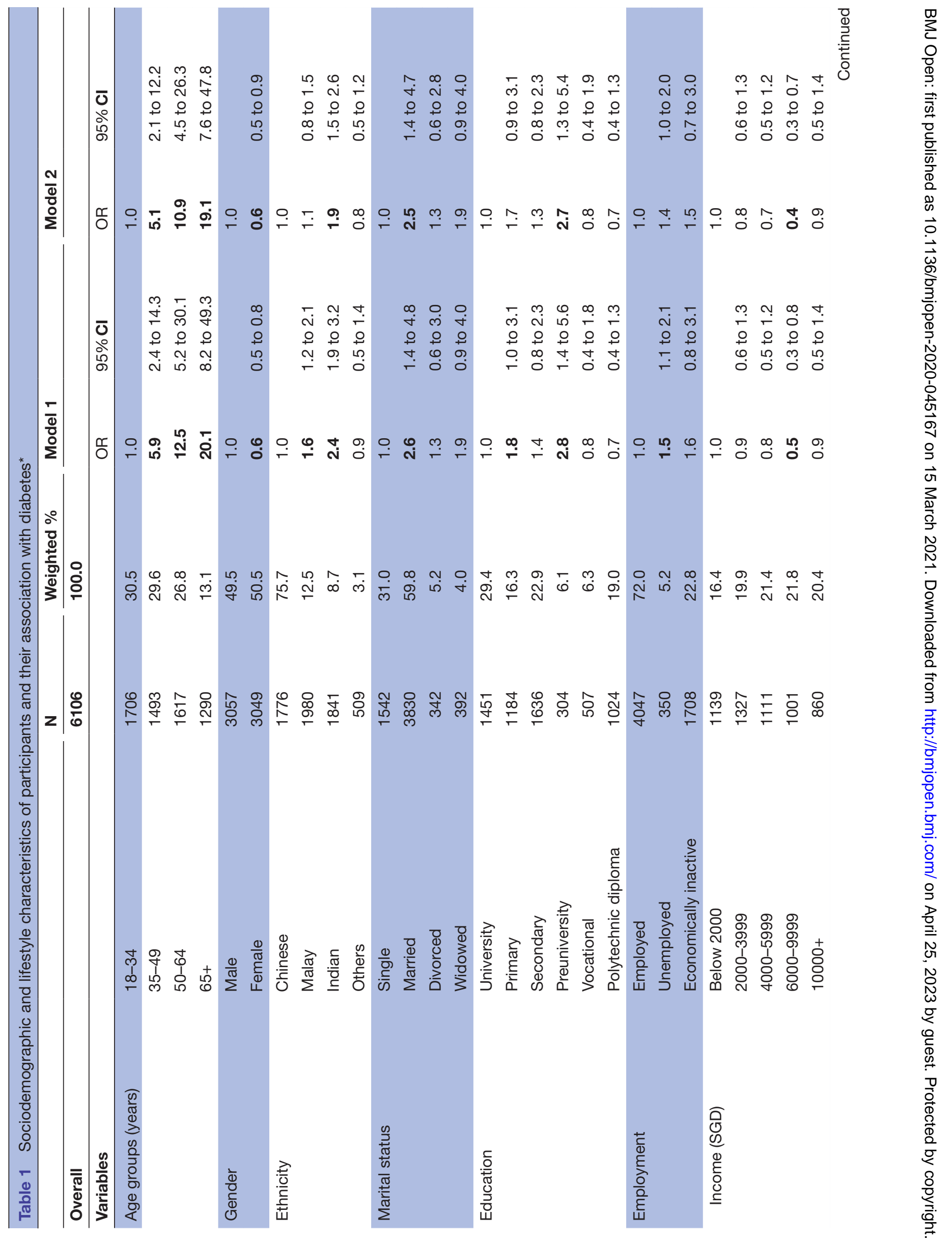




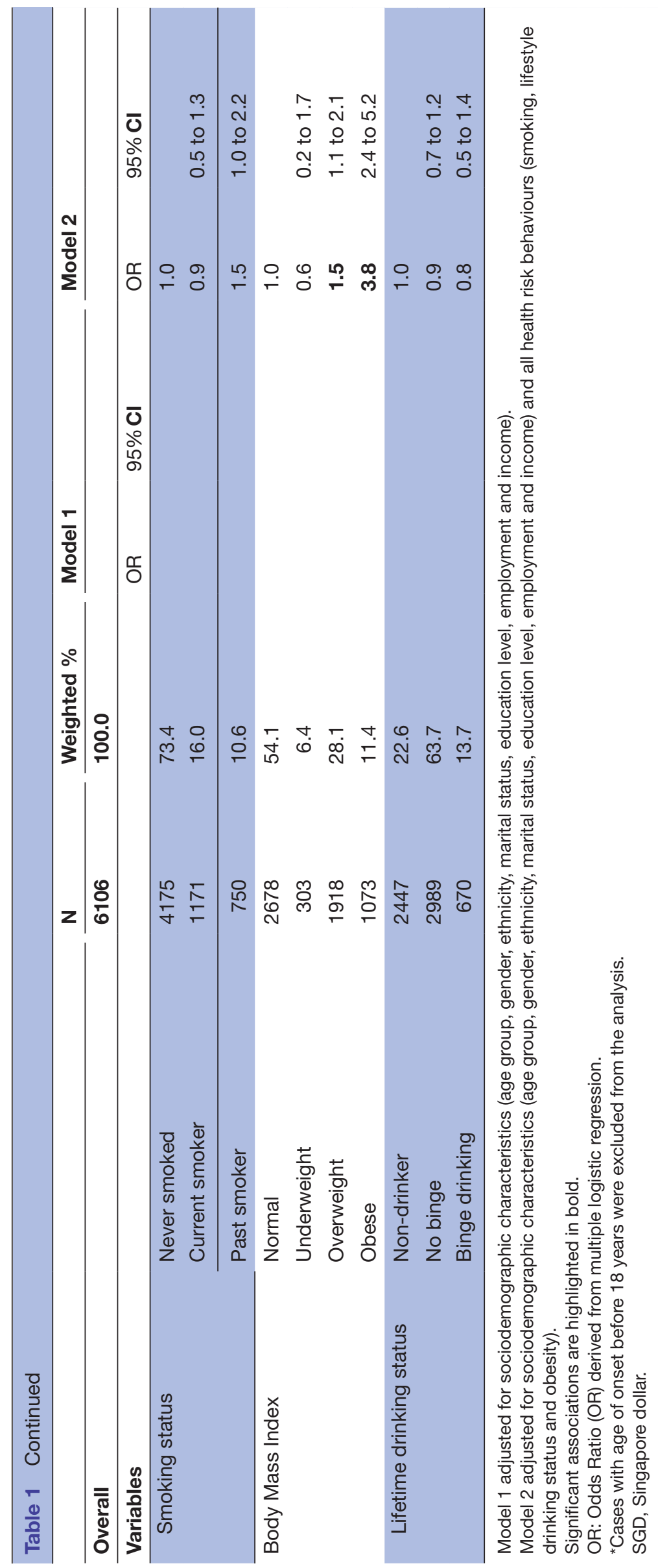

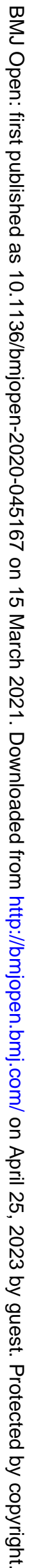


Table 2 Association between type/frequencies of ACEs and diabetest in the overall sample

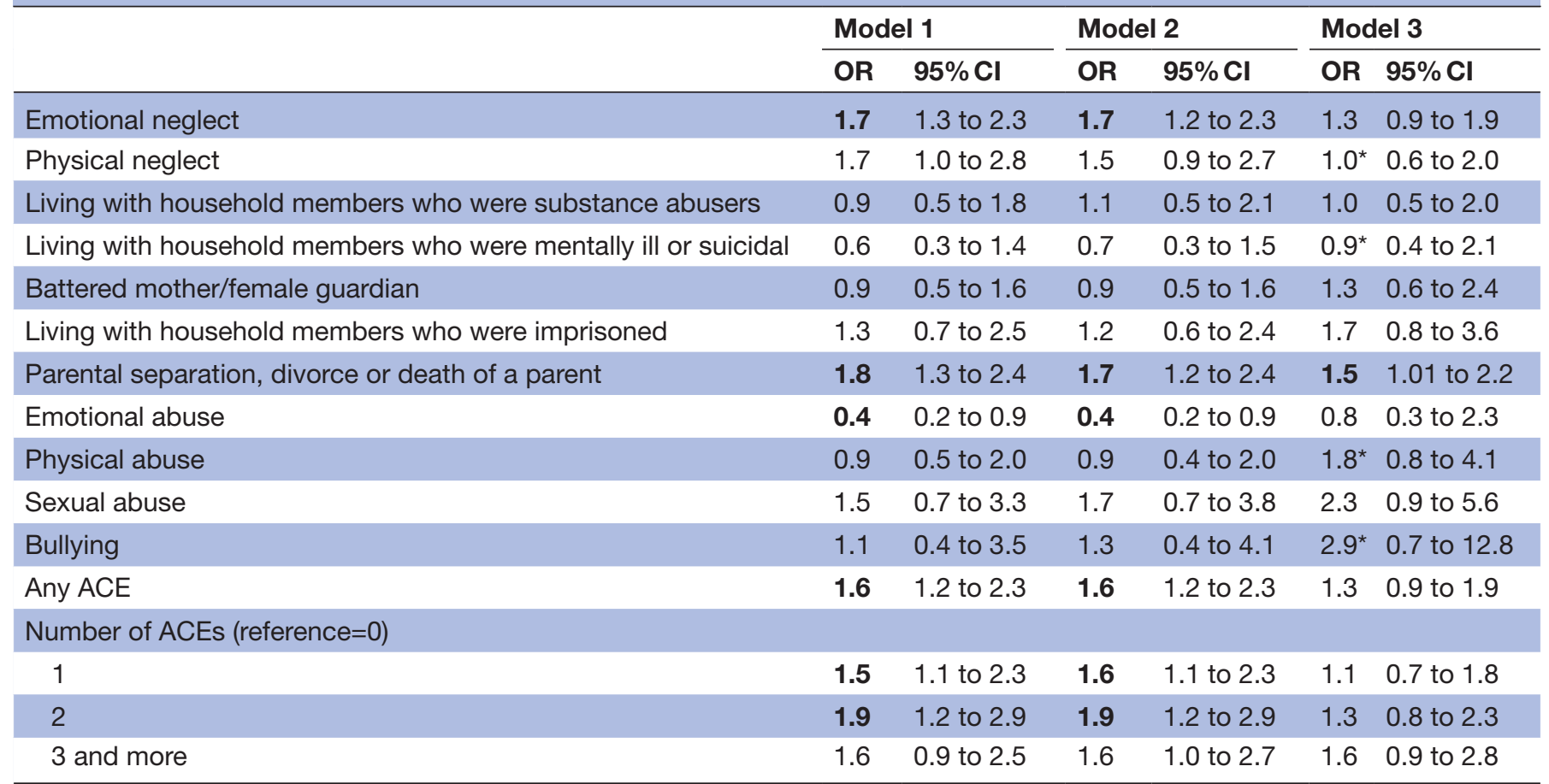

Model 1: crude OR.

Model 2: ORs were adjusted for health risk behaviours (ie, BMI, drinking and smoking).

Model 3: ORs were adjusted for sociodemographic factors (ie, age, gender, ethnicity, marital status, education and income) and health risk behaviours (ie, BMI, drinking and smoking).

Significant associations are highlighted in bold.

*Significant interaction effect between age group and ACE.

†Cases with age of onset before 18 years were excluded from the analysis.

ACE, adverse childhood experience; BMI, Body Mass Index.

associated with diabetes. In contrast, ACEs were not significantly associated with higher odds of diabetes in the older age group.

Associations with specific ACEs vary widely across studies. Monnat and Chandler ${ }^{47}$ found that physical abuse, sexual abuse and exposure to domestic violence increased the odds of diabetes in adult life, while Rich-Edwards $e t a l^{28}$ found that physical and sexual abuse in childhood and adolescence were associated with risk of type 2 diabetes among adult women. Longitudinal data from the 1979 National Longitudinal Survey of Youth found that type 2 diabetes was associated with parental death in women. ${ }^{48}$ On the other hand, Widom et al, ${ }^{49}$ using data from court records, found that physical abuse and neglect (largely physical neglect) were associated with diabetes in middle adulthood.

Several reasons have been suggested to explain the association of ACEs with the development of diabetes and other chronic physical conditions. ACEs negatively impact neurodevelopment especially that of the hypothalamic-pituitary-adrenal axis, ${ }^{50}$ cause an elevated level of triglycerides, free fatty acids and glucose in the blood, ${ }^{23}$ and they result in the elevation of inflammatory markers, all of which have been postulated to be involved in the development of chronic illnesses including diabetes. ${ }^{51}$
In the older population, that is, those more than 50 years of age, living with household members who were mentally ill or suicidal and emotional abuse were both less likely to have diabetes. We are currently unable to explain this association. This suggests that while age remains one of the most significant risk factors for diabetes, ACEs are an important risk factor for diabetes among those belonging to the younger age groups. Interestingly, the age of onset of diabetes was significantly earlier in the age group of 18-49 years, compared with the age of onset in those older than 50 years, which raises the possibility that ACEs may be linked to an earlier onset of diabetes and thus are more strongly associated with diabetes in those belonging to the younger age group. A study examining the association of ACEs with chronic diseases found that young adults who had experienced four or more ACEs (compared with no ACEs) were at a much greater risk of both pre-diabetes and diabetes. The incident rate ratios (IRRs) for diabetes and pre-diabetes were highest in the youngest age group and lower in the older age groups. The authors suggested that the attenuated IRR in the older age groups could be due to the increase in other risk factors which an individual may be exposed to regardless of any history of ACE. ${ }^{52}$ Survival bias could also account for the lower association between ACE and diabetes 
Table 3 Association between type and frequencies of ACEs and diabetes ${ }^{\star}$ after controlling for health risk behaviours and sociodemographic factors

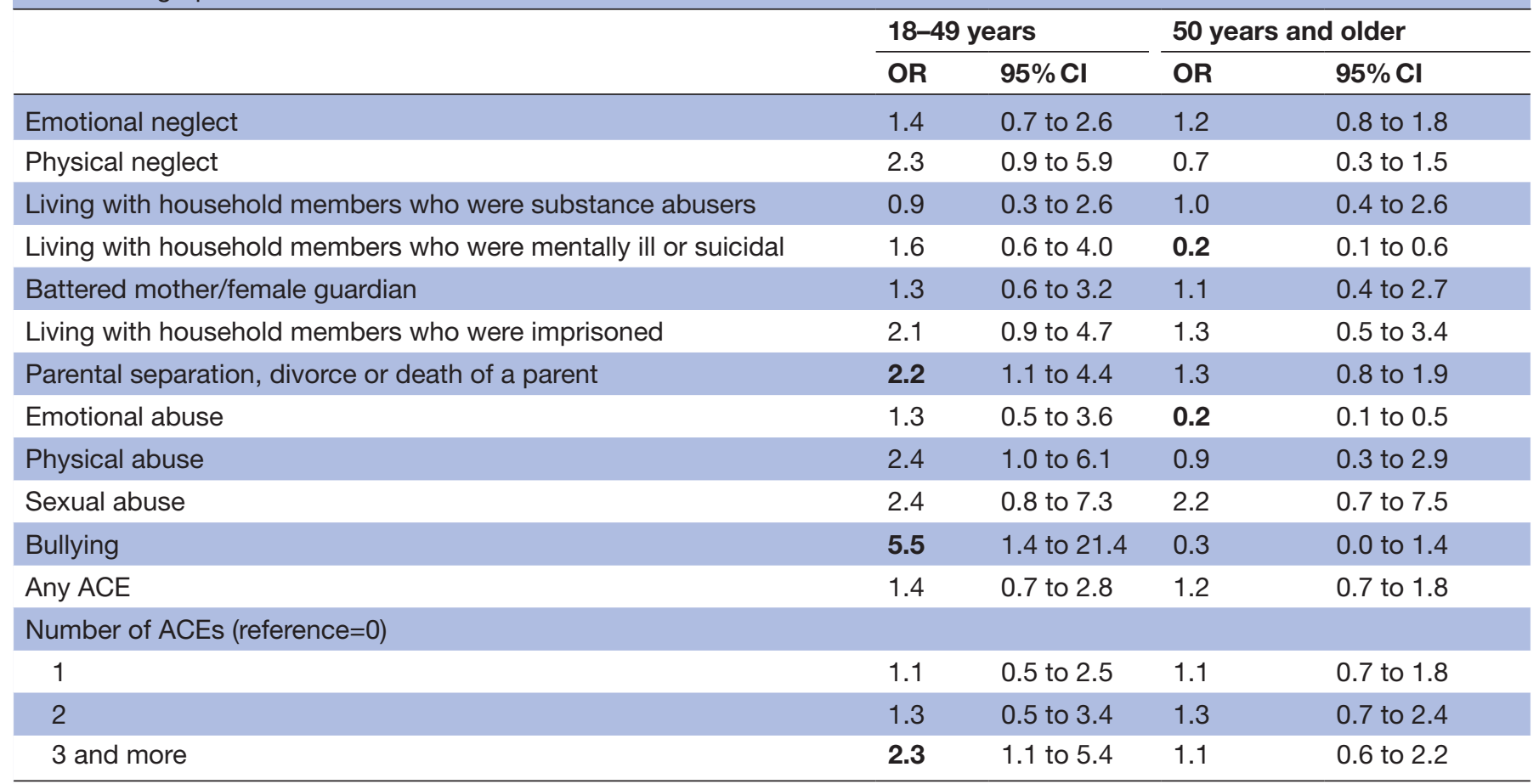

ORs were adjusted for sociodemographic factors (ie, age, gender, ethnicity, marital status, education and income) and health risk behaviours (ie, BMI, drinking and smoking).

Significant associations are highlighted in bold.

${ }^{*}$ Cases with age of onset before 18 years were excluded from the analysis.

ACE, adverse childhood experience; BMI, Body Mass Index.

among the older age group in our study, as other studies have observed a reduction in life expectancy among those exposed to a high burden of ACEs. ${ }^{53}$

Several studies have highlighted that health risk behaviours which are associated with ACEs may explain poor outcomes such as diabetes in this group. However, on adjusting for health risk behaviours, the association between ACEs and diabetes did not change significantly for the overall sample in our study. These results are similar to that of Kreatsoulas $e t a l,{ }^{54}$ who found that when they tested the association of ACEs with diabetes through health behaviours which are known risk factors such as obesity, the relationship did not attenuate significantly. The authors suggested that ACEs may operate on an independent and potent pathway early in life.

Few studies have examined the impact of ACEs among those with diabetes. Our study, in line with our hypothesis, found that those with ACE and diabetes were more likely to be associated with MDD, and lower HRQoL. Stress and inflammatory mechanisms have been suggested to explain the association between diabetes and depres$\operatorname{sion}^{55}$; it therefore follows that those with diabetes and ACEs would have greater cumulative stress that could lead to a higher prevalence of depression in this group. On the other hand, a mediation analysis suggested that ACEs were indirectly associated with an increased risk of diabetes in adulthood via depressive symptoms. ${ }^{24}$ Corso et $a l^{33}$ have reported lower HRQoL among those with ACEs. Thus, ACEs alone or in combination with depression could have led to a lower HRQoL among those with diabetes and ACES.

However, in terms of resource use, the results did not support our hypothesis. Our study, unlike that of Hargreaves et $a l,{ }^{56}$ who reported greater use of emergency rooms and doctor office visits with increasing ACEs as compared with those without any ACEs, did not find increased resource use among those with diabetes and ACEs. Koball et al, ${ }^{12}$ examining the impact of ACEs on resource use, found that patients with higher ACEs were more likely to miss appointments or 'late-cancelled' appointments. Thus, while resource use may be low, there would be an enormous cost to the healthcare system. The lack of significant association of those with diabetes and ACEs may thus be due to the fact that we did not examine it in those with high ACEs (current analysis included those with at least one ACE) or it could be that while those with ACEs needed services, they did not attend them.

The prevalence of diabetes in our study sample was 9.7\%. Sociodemographic factors and health behaviours including older age, Indian ethnicity, as well as being overweight and obese were associated with diabetes, whereas female gender was associated with a lower prevalence of diabetes. These findings are similar to the prevalence figures of $9.1 \%$ of diabetes in Singapore as reported 
Table 4 Associations between combinations of diabetes and adverse childhood events with major depressive disorder, resource use, and health-related quality of life domains

\begin{tabular}{|c|c|c|c|c|c|}
\hline & $\begin{array}{l}\text { (A) Diabetes with } \\
\text { ACE }\end{array}$ & $\begin{array}{l}\text { (B) Diabetes without } \\
\text { ACE }\end{array}$ & & & \\
\hline & $(\mathrm{n}=350)$ & $(n=137)$ & A versus B (refer & ence $)^{*}$ & \\
\hline & n (\%) & n (\%) & OR & $95 \% \mathrm{Cl}$ & $P$ value \\
\hline Major depressive disorder & $15(3.7)$ & $1(0.3)$ & 19.7 & 2.1 to 187.4 & 0.010 \\
\hline \multicolumn{6}{|l|}{ Resource use } \\
\hline Primary care doctor & $172(44.8)$ & $66(37.2)$ & 1.1 & 0.5 to 2.2 & 0.855 \\
\hline Restructured hospital doctor & $105(27.0)$ & $40(26.7)$ & 1.3 & 0.6 to 2.6 & 0.513 \\
\hline \multirow[t]{2}{*}{ Private hospital/clinic doctors } & $107(30.3)$ & $36(39.3)$ & 0.8 & 0.3 to 1.7 & 0.483 \\
\hline & Mean (SD) & Mean (SD) & Beta coefficient & $95 \% \mathrm{Cl}$ & $P$ value \\
\hline Number of hospitalisations & $0.5(4.2)$ & $0.3(3.2)$ & 0.2 & -0.5 to 0.8 & 0.607 \\
\hline \multicolumn{6}{|l|}{ Quality of life domains } \\
\hline Physical functioning & $51.0(11.8)$ & $51.1(11.2)$ & 0.4 & -2.0 to 2.9 & 0.736 \\
\hline Role-physical & $52.6(9.6)$ & $54.8(7.6)$ & -1.8 & -3.8 to 0.2 & 0.075 \\
\hline Bodily pain & $27.3(10.7)$ & $24.5(8.5)$ & 2.1 & -0.3 to 4.6 & 0.087 \\
\hline General health & $48.2(12.8)$ & $45.0(11.9)$ & 2.6 & -0.7 to 6.0 & 0.120 \\
\hline Vitality & $53.8(11.8)$ & $57.4(10.0)$ & -3.5 & -6.2 to -0.8 & 0.014 \\
\hline Social functioning & $53.7(9.3)$ & $55.4(6.1)$ & -1.3 & -3.3 to 0.7 & 0.208 \\
\hline Role-emotional & $53.3(8.9)$ & $55.5(4.4)$ & -1.7 & -3.0 to -0.5 & 0.011 \\
\hline Mental health & $55.1(10.4)$ & $58.8(7.2)$ & -4.0 & -6.2 to -1.8 & $<0.001$ \\
\hline PCS & $42.0(7.2)$ & $40.0(5.9)$ & 2.0 & 0.5 to 3.4 & 0.009 \\
\hline MCS & $57.5(10.6)$ & $61.6(6.1)$ & -3.9 & -5.8 to -1.9 & $<0.001$ \\
\hline
\end{tabular}

Significant associations are highlighted in bold.

${ }^{\star}$ Regression analyses were conducted after controlling for sociodemographic factors (ie, age, gender, ethnicity, marital status, education and income) and health risk behaviours (ie, BMI, smoking and drinking).

ACE, adverse childhood experience; BMI, Body Mass Index; MCS, mental component summary; PCS, physical component summary.

by the WHO, which also identified overweight, obesity and physical inactivity as significant risk factors. ${ }^{57}$ Older age and Indian ethnicity have similarly been identified as risk factors for diabetes in Singapore. ${ }^{58}$ These results add support to the validity of the study methodology and our data.

\section{Limitations}

Some limitations of the study need to be noted so that the gaps can be addressed in future studies. Data on ACEs were captured retrospectively; there is the possibility of recall bias, and given the sensitivity of the questions, there could have been a tendency to under-report the experience. However, we have tried to minimise this by assuring the participants of the confidential nature of the study and that no participant identifiers would be collected. Furthermore, the ACE questionnaire was selfadministered by participants to reduce under-reporting as much as possible. Some of the participants refused to complete the ACE questionnaire, but the participation rates were comparable to other studies. ${ }^{42} 59$ The study did not assess the severity, age of onset and duration of ACEs, which may have an impact on the development of diabetes. ${ }^{28}{ }^{60}$ Diagnosis of diabetes was also self-reported, and thus we would have missed out those who had not been given a clinical diagnosis of diabetes. The questions in our study did not differentiate between type 1 and type 2 diabetes. Type 1 diabetes has an autoimmune aetiology and risk factors are different from those of type 2 diabetes. Including those with type 1 diabetes could have led to weakening of the association between ACEs and diabetes in our study as ACEs are not likely to be associated with type 1 diabetes. ${ }^{61}$ However, onset of type 1 diabetes is rare in adults. The current analysis only included those with age of onset of diabetes after 18 years, and diabetes is therefore likely to predominantly be type 2 diabetes. Local data have established a high prevalence of diabetes among Indians. ${ }^{35}$ However, the survey questionnaire was not available in Tamil, which is one of the official languages of Singapore and spoken by a large proportion of Indians. This may have affected the participation of people of Indian ethnicity and the prevalence of diabetes established in the study. However, the authors believe that this would not have affected the study significantly as English literacy is high in this group. A study by 
Mathew et $a l^{62}$ found that $86 \%$ of Indians above the age of 65 years and $91 \%$ of those in the age range of 51-65 years can speak English well or very well. Lastly, the crosssectional design limits assertions regarding the sequence of causal events, but this was overcome to a significant extent by excluding those with onset of diabetes before 18 years of age.

\section{CONCLUSIONS}

Our study establishes an association between ACEs and diabetes in a multiethnic urban population of a developed Asian country. Among those with diabetes, presence of ACEs worsens outcomes in terms of comorbidity with MDD and poorer HRQoL. Our findings highlight the need to address ACEs across society to improve health outcomes for the population. A multiagency effort is needed across both health and social care sectors to screen, prevent and provide early care for those who have experienced ACEs. However, we must be cognisant that advancing age and BMI are other important factors associated with diabetes. Thus, efforts to promote regular exercise and healthy lifestyles both in the population and among those with diabetes must continue, along with better identification of and communication about ACEs within the healthcare setting. We need to develop traumainformed methods for screening and treating childhood trauma. We also need to create more awareness of both the prevalence and impact of ACEs not just among mental health providers but also among those treating chronic diseases. A collaborative care approach to treat those with chronic diseases and ACEs that addresses their medical, mental health and trauma-related issues will improve outcomes for the person and the society as a whole.

Contributors SAC, MS and JAV were involved in the planning of the study and provided advice and guidance throughout the conduct of the study, and manuscript writing. SC, RS, AJ and LSES were involved in the conduct of the study. EA conducted the statistical analyses. RVD guided the data analysis. RVD and WLC provided expert opinion. All authors gave critical comment throughout the writing process. All authors were responsible for the conceptualisation and design of the project. All authors approved the final version to be published.

Funding The study was funded by the Ministry of Health Singapore and Temasek Foundation (grant number: not applicable).

Competing interests None declared.

Patient consent for publication Not required.

Ethics approval The research was approved by the National Healthcare Group Domain Specific Review Board (2015/01035) and was given hospital institutional approval. Written informed consent was obtained from all participants and parents or legally acceptable representatives of those aged below 21 years.

Provenance and peer review Not commissioned; externally peer reviewed.

Data availability statement Data are available upon reasonable request. Raw data from this study are currently not publicly available but can be made available upon reasonable request from the corresponding author.

Supplemental material This content has been supplied by the author(s). It has not been vetted by BMJ Publishing Group Limited (BMJ) and may not have been peer-reviewed. Any opinions or recommendations discussed are solely those of the author(s) and are not endorsed by BMJ. BMJ disclaims all liability and responsibility arising from any reliance placed on the content. Where the content includes any translated material, BMJ does not warrant the accuracy and reliability of the translations (including but not limited to local regulations, clinical guidelines, terminology, drug names and drug dosages), and is not responsible for any error and/or omissions arising from translation and adaptation or otherwise.

Open access This is an open access article distributed in accordance with the Creative Commons Attribution Non Commercial (CC BY-NC 4.0) license, which permits others to distribute, remix, adapt, build upon this work non-commercially, and license their derivative works on different terms, provided the original work is properly cited, appropriate credit is given, any changes made indicated, and the use is non-commercial. See: http://creativecommons.org/licenses/by-nc/4.0/.

ORCID iDs

Mythily Subramaniam http://orcid.org/0000-0003-4530-1096

Sherilyn Chang http://orcid.org/0000-0001-9716-4543

\section{REFERENCES}

1 Felitti VJ, Anda RF, Nordenberg D, et al. Relationship of childhood abuse and household dysfunction to many of the leading causes of death in adults. The adverse childhood experiences (ACE) study. Am J Prev Med 1998;14:245-58.

2 Sacks V, Murphey D, Moore K. Adverse childhood experiences: national and state-level prevalence. Bethesda, Maryland: Child Trends, 2014

3 Bellis MA, Hughes K, Leckenby N, et al. National household survey of adverse childhood experiences and their relationship with resilience to health-harming behaviors in England. BMC Med 2014;12:72.

4 Nurius PS, Logan-Greene P, Green S. Adverse childhood experiences (ACE) within a social disadvantage framework: distinguishing unique, cumulative, and moderated contributions to adult mental health. $J$ Prev Interv Community 2012;40:278-90.

5 Kelly-Irving M, Lepage B, Dedieu D, et al. Childhood adversity as a risk for cancer: findings from the 1958 British birth cohort study. BMC Public Health 2013;13:767.

6 Su S, Wang X, Pollock JS, et al. Adverse childhood experiences and blood pressure trajectories from childhood to young adulthood: the Georgia stress and heart study. Circulation 2015;131:1674-81.

7 Lippard ETC, Nemeroff CB. The devastating clinical consequences of child abuse and neglect: increased disease vulnerability and poor treatment response in mood disorders. Am J Psychiatry 2020;177:20-36.

8 Brown DW, Anda RF, Tiemeier H. Adverse childhood experiences and the risk of premature mortality. Am J Prev Med 2009;37:389-96.

9 Lee C, White HR. Effects of childhood maltreatment on violent injuries and premature death during young adulthood among urban high-risk men. Arch Pediatr Adolesc Med 2012;166:814-20.

10 Bellis MA, Hughes K, Hardcastle K. The impact of adverse childhood experiences on health service use across the life course using a retrospective cohort study. J Health Serv Res Policy 2017;22:168-77.

11 Mercado RC, Wiltsey-Stirman S, Iverson KM. Impact of childhood abuse on physical and mental health status and health care utilization among female veterans. Mil Med 2015;180:1065-74.

12 Koball AM, Rasmussen C, Olson-Dorff D, et al. The relationship between adverse childhood experiences, healthcare utilization, cost of care and medical comorbidities. Child Abuse Negl 2019;90:120-6.

13 Chartier MJ, Walker JR, Naimark B. Childhood abuse, adult health, and health care utilization: results from a representative community sample. Am J Epidemiol 2007;165:1031-8.

14 Saeedi P, Petersohn I, Salpea P, et al. Global and regional diabetes prevalence estimates for 2019 and projections for 2030 and 2045: Results from the International Diabetes Federation Diabetes Atlas, $9^{\text {th }}$ edition. Diabetes Res Clin Pract 2019;157:107843.

15 NCD Risk Factor Collaboration (NCD-RisC). Worldwide trends in diabetes since 1980: a pooled analysis of 751 population-based studies with 4.4 million participants. Lancet 2016;387:1513-30.

16 International Diabetes Federation. IDF diabetes atlas, 9th ed. Brussel, Belgium: International Diabetes Federation, 2019. https:// diabetesatlas.org/upload/resources/2019/IDF_Atlas_9th_Edition_ 2019.pdf

17 Huang H, Yan P, Shan Z, et al. Adverse childhood experiences and risk of type 2 diabetes: a systematic review and meta-analysis. Metabolism 2015;64:1408-18.

18 Lynch L, Waite R, Davey MP. Adverse childhood experiences and diabetes in adulthood: support for a collaborative approach to primary care. Contemp Fam Ther 2013;35:639-55.

19 Huffhines L, Noser A, Patton SR. The link between adverse childhood experiences and diabetes. Curr Diab Rep 2016;16:54.

20 Jakubowski KP, Cundiff JM, Matthews KA. Cumulative childhood adversity and adult cardiometabolic disease: a meta-analysis. Health Psychol 2018;37:701-15. 
21 Chan O, Inouye K, Riddell MC, et al. Diabetes and the hypothalamopituitary-adrenal (HPA) axis. Minerva Endocrinol 2003;28:87-102.

22 Rao U, Hammen C, Ortiz LR, et al. Effects of early and recent adverse experiences on adrenal response to psychosocial stress in depressed adolescents. Biol Psychiatry 2008;64:521-6.

23 Diz-Chaves Y, Gil-Lozano M, Toba L, et al. Stressing diabetes? the hidden links between insulinotropic peptides and the HPA axis. $J$ Endocrinol 2016;230:R77-94.

24 Deschênes SS, Graham E, Kivimäki M, et al. Adverse childhood experiences and the risk of diabetes: examining the roles of depressive symptoms and cardiometabolic dysregulations in the Whitehall II cohort study. Diabetes Care 2018;41:2120-6.

25 Baumeister D, Akhtar R, Ciufolini S, et al. Childhood trauma and adulthood inflammation: a meta-analysis of peripheral C-reactive protein, interleukin- 6 and tumour necrosis factor- $\alpha$. Mol Psychiatry 2016;21:642-9.

26 Yang B-Z, Zhang H, Ge W, et al. Child abuse and epigenetic mechanisms of disease risk. Am J Prev Med 2013;44:101-7.

27 Suderman M, Borghol N, Pappas JJ, et al. Childhood abuse is associated with methylation of multiple loci in adult DNA. BMC Med Genomics 2014;7:1-12.

28 Rich-Edwards JW, Spiegelman D, Lividoti Hibert EN, et al. Abuse in childhood and adolescence as a predictor of type 2 diabetes in adult women. Am J Prev Med 2010;39:529-36.

29 Danese A, Tan M. Childhood maltreatment and obesity: systematic review and meta-analysis. Mol Psychiatry 2014;19:544-54.

30 Davis CR, Dearing E, Usher N, et al. Detailed assessments of childhood adversity enhance prediction of central obesity independent of gender, race, adult psychosocial risk and health behaviors. Metabolism 2014;63:199-206.

31 Farr OM, Ko B-J, Joung KE, et al. Posttraumatic stress disorder, alone or additively with early life adversity, is associated with obesity and cardiometabolic risk. Nutr Metab Cardiovasc Dis 2015;25:479-88.

32 Danese A, Moffitt TE, Harrington $\mathrm{H}$, et al. Adverse childhood experiences and adult risk factors for age-related disease: depression, inflammation, and clustering of metabolic risk markers. Arch Pediatr Adolesc Med 2009;163:1135-43.

33 Corso PS, Edwards VJ, Fang X, et al. Health-Related quality of life among adults who experienced maltreatment during childhood. Am J Public Health 2008;98:1094-100.

34 Subramaniam M, Abdin E, Seow E, et al. Prevalence, sociodemographic correlates and associations of adverse childhood experiences with mental illnesses: results from the Singapore mental health study. Child Abuse Negl 2020;103:104447.

35 Ministry of Health. National health survey, 2010. Available: https:// www.moh.gov.sg/content/dam/moh_web/Publications/Reports/ 2011/NHS2010\%20-\%20low\%20res.pdf

36 Ministry of Health. Executive summary on national population health survey 2016/17, 2017. Available: https://www.moh.gov.sg/docs/ librariesprovider5/resources-statistics/reports/executive-summarynphs-2016_17.pdf

37 Subramaniam M, Abdin E, Vaingankar JA, et al. Tracking the mental health of a nation: prevalence and correlates of mental disorders in the second Singapore mental health study. Epidemiol Psychiatr Sci 2020;29:E29.

38 Kessler RC, Ustün TB. The world mental health (WMH) survey initiative version of the world Health organization (who) composite international diagnostic interview (CIDI). Int J Methods Psychiatr Res 2004;13:93-121.

39 Chong SA, Abdin E, Nan L, et al. Prevalence and impact of mental and physical comorbidity in the adult Singapore population. Ann Acad Med Singap 2012;41:105-14.

40 World Health Organization. Adverse childhood experiences international questionnaire adverse childhood experiences international questionnaire (ACE-IQ), who, Geneva, 2018. Available: https://www.who.int/violence_injury_prevention/ violence/activities/adverse_childhood_experiences/en/ [Accessed 3 March 2021]
41 Bruffaerts R, Demyttenaere K, Borges G, et al. Childhood adversities as risk factors for onset and persistence of suicidal behaviour. $\mathrm{Br} J$ Psychiatry 2010;197:20-7.

42 Hughes K, Bellis MA, Hardcastle KA, et al. The effect of multiple adverse childhood experiences on health: a systematic review and meta-analysis. Lancet Public Health 2017;2:e356-66.

43 Scott KM, Von Korff M, Angermeyer MC et al. Association of childhood adversities and early-onset mental disorders with adult-onset chronic physical conditions. Arch Gen Psychiatry 2011;68:838-44.

44 World Health Organization. WHO technical report series 894; obesity: preventing and managing the global epidemic, 2000. Available: http://www.who.int/nutrition/publications/obesity/WHO_TRS_894/en/

45 Ware J, Kosinski M, Keller SD. A 12-Item short-form health survey: construction of scales and preliminary tests of reliability and validity. Med Care 1996;34:220-33.

46 Beecham J, Knapp M. Costing psychiatric interventions. In: Thornicroft G, Brewin CR, Wing J, eds. Measuring mental health needs. London: Gaskell, 1992: 163-83.

47 Monnat SM, Chandler RF. Long term physical health consequences of adverse childhood experiences. Sociol Q 2015;56:723-52.

48 Lown EA, Lui CK, Karriker-Jaffe K, et al. Adverse childhood events and risk of diabetes onset in the 1979 national longitudinal survey of youth cohort. BMC Public Health 2019;19:1007.

49 Widom CS, Czaja SJ, Bentley T, et al. A prospective investigation of physical health outcomes in abused and neglected children: new findings from a 30-year follow-up. Am J Public Health 2012;102:1135-44.

50 Kalmakis KA, Meyer JS, Chiodo L, et al. Adverse childhood experiences and chronic hypothalamic-pituitary-adrenal activity. Stress 2015;18:446-50.

51 Heard-Garris N, Davis MM, Estabrook R, et al. Adverse childhood experiences and biomarkers of inflammation in a diverse cohort of early school-aged children. Brain Behav Immun Health 2020;1:100006.

52 Sonu S, Post S, Feinglass J. Adverse childhood experiences and the onset of chronic disease in young adulthood. Prev Med 2019;123:163-70.

53 Brown DW, Anda RF, Tiemeier H, et al. Adverse childhood experiences and the risk of premature mortality. Am J Prev Med 2009;37:389-96.

54 Kreatsoulas C, Fleegler EW, Kubzansky LD, et al. Young adults and adverse childhood events: a potent measure of cardiovascular risk. Am J Med 2019;132:605-13.

55 Bădescu SV, Tătaru C, Kobylinska L, et al. The association between diabetes mellitus and depression. J Med Life 2016;9:120-5.

56 Hargreaves MK, Mouton CP, Liu J, et al. Adverse childhood experiences and health care utilization in a low-income population. $J$ Health Care Poor Underserved 2019;30:749-67.

57 World Health Organization. Global report on diabetes. Geneva, Switzerland: World Health Organization, 2016.

58 National Registry of Diseases Office. Information paper on diabetes in Singapore, 2011. Available: https://www.nrdo.gov.sg/docs/ librariesprovider3/Publications---Kidney-Failure/info_paper diabetes_2011nov.pdf [Accessed 3 March 2021]

59 Häuser W, Schmutzer G, Brähler E, et al. Maltreatment in childhood and adolescence: results from a survey of a representative sample of the German population. Dtsch Arztebl Int 2011;108:287-94.

60 Karavanaki K, Tsoka E, Liacopoulou M, et al. Psychological stress as a factor potentially contributing to the pathogenesis of type 1 diabetes mellitus. J Endocrinol Invest 2008;31:406-15.

61 Bengtsson J, Byberg S, Carstensen B, et al. Accumulation of childhood adversities and type 1 diabetes risk: a register-based cohort study of all children born in Denmark between 1980 and 2015 Int J Epidemiol 2020;49:1604-13.

62 Mathew M, Tay M, Selvarajen S, et al. Language proficiency, identity \& management: results from the IPS survey on race, religion \& language. IPS Exchange, Number 15 June 2020, 2020. Available: https://lkyspp.nus.edu.sg/docs/default-source/ips/ips-exchangeseries-15.pdf 